\title{
Sexual Function and Factors Affecting Menopause: A Systematic Review
}

\author{
Mohammad Heidari ${ }^{1}$, Mansureh Ghodusi ${ }^{2}$, Parvin Rezaei ${ }^{3}$, Shokouh Kabirian Abyaneh ${ }^{4}$, \\ Ehsan Heidari Sureshjani ${ }^{5}$, Rahim Ali Sheikhi ${ }^{6}$ \\ ${ }^{1}$ Community-Oriented Nursing Midwifery Research Center, Shahrekord University of Medical Sciences, Shahrekord, Iran, ${ }^{2}$ Young Researchers \\ and Elite Club, Abadeh Branch, Islamic Azad University, Abadeh, Iran, ${ }^{3}$ Department of Infectious Disease and Tropical Medicine, Shariati \\ Hospital, Tehran University of Medical Sciences, Tehran, Iran, ${ }^{4}$ Department of Nursing, Shahid Beheshti University of Medical Sciences, \\ Tehran, Iran, ${ }^{5}$ Prehospital Emergency Care, Disaster and Emergency Medical Management Center, ShahreKord University of Medical Sciences, \\ Shahrekord, Iran, ${ }^{6}$ Health Management and Economics Research Center, Iran University of Medical Sciences, Tehran, Iran
}

The purpose of this review study is to evaluate sexual function and its effective factors in menopause. A systematic review was conducted according to the Preferred Reporting Items for Systematic Reviews and Meta-Analyses (PRISMA) guidelines. The key words of "menopause," "postmenopause," "postmenopausal," "premenopause," "pre-menopausal period," "sexual function," "sexual health," "sexuality," "sexual and gender disorders," "sexual development," "sexual dysfunction," "sexual disorders," "sexual behavior and "sexual activity" were used in combination with the Boolean operators OR and AND. After reviewing the selected articles, 27 papers were selected based on the criteria for entering the study and the goals set. The results of the reviewed articles showed that, in the physical domain, the factors affecting sexual function can be mentioned, age, hormonal changes, medical problems and reproductive history. Sexual disorders in menopause can be affected by some of the individual and social characteristics and psychological problems. Considering the fact that many psychological and social injuries occur in this period following sexual disorders; therefore, policies and programs for improving the quality of life of women in menopause should be aimed at eliminating sexual dysfunction, correcting attitudes and negative emotions and help to women for more comfortable in menopause. (J Menopausal Med 2019;25:15-27)

Key Words: Sexual health · Sexual dysfunctions · Sexual behavior · Menopause

\section{Introduction}

Menopause is one of the evolutionary stages that all women experience through aging, and this phenomenon exposes them to massive changes. ${ }^{1,2}$ Reducing estrogen levels in early stages of menopause period leads to a wide range of symptoms which can affect women's physical, mental and sexual health and affect work, social activities, mood, communication with others, life pleasure, and ultimately the quality of their life. ${ }^{3}$ In fact, women during menopause experience a transitional period that if a person feels that he is not able to change the structure of her life, there may be a developmental crisis in her life, and the physiological changes of this period may have a profound effect on her personal feelings and satisfaction with her life. ${ }^{4,5}$ The most comprehensive talk of menopause is menstruation, following the decline in ovarian activity at the end of women's fertility period, and short and medium term effects such as

Received: September 21, 2018 Revised: November 1, 2018 Accepted: November 16, 2018

Address for Correspondence: Rahim Ali Sheikhi, Health Management and Economics Research Center, Iran University of Medical Sciences, No 6, Rashid Yasemi St., Vali-e-asr Ave., Tehran 1995614111, Iran

Tel: +98-913-2810140, Fax: +98-21-88883334, E-mail: rahim.shaykhi94@gmail.com

ORCID: http://orcid.org/0000-0002-2034-8624

Copyright (c) 2019 by The Korean Society of Menopause

(c) This is an Open Access article distributed under the terms of the Creative Commons Attribution Non-Commercial License (http://creativecommons.org/licenses/by-nc/4.0/). 
vasomotor disorders, urinary symptoms, atrophic vaginitis, and sexual disorders, as well as long-term effects such as osteoporosis and cardio-vascular disorders, along with many evolutionary, physical, emotional and social abnormalities. ${ }^{6}$ Sexuality and sexual function is regarded as one of the issues, which can be considered in this regard. A set of psychosocial aspects such as sexual stimulation and sexual desire are called sexual function. ${ }^{7}$ Sexuality is defined as a physical, emotional, psychological, and social welfare state associated with sexual desires, not just lack of disease, dysfunction, or disability. At this stage of life, women will face many problems and complications from the lack of sex hormones. ${ }^{8}$ Sexual relationships are one of the most important factors affecting the happiness of marital life, which, if not convincing, leads to a feeling of failure, defeat, and lack of security. ${ }^{9}$ The most significant causes of sexual disorders in this period are physiological changes of body, psychological problems and lack of sexual knowledge, ${ }^{10}$ which cause the sensitive period of menopause to become very risky, because sexual disorders lead to the destruction of the mental health of the family by creating or intensifying the psychological problems reciprocally. In this regard, many of the aggressions, controversies, and monitoring and control of couples in each other's activities can be linked to these disorders. ${ }^{9,10}$ Recognizing and studying human sexual behaviors is one of the most important public health issues, especially mental health. ${ }^{11}$ The World Health Organization considers sexual hygiene as an integrity and coordination among mind, body, and soul in order to promote human's social and intellectual aspects in her or his way through personality promotion, leading to communication and love. Therefore, any disorder leading to inconsistency and as a consequence of dissatisfaction with the sexual relationship can cause sexual dysfunction. ${ }^{12}$ The prevalence of sexual dysfunction among all women is estimated to be $25 \%$ to $63 \%$. This outbreak is higher in women with menopause and reaches from $68 \%$ to 86.5\%. Sexuality is not exclusively a physical phenomenon, but it relies on the individual's lifestyle, a deep and comprehensive grasp of someone from herself as a human being, the attitudes of others towards her and other factors.

Sexuality is more involved than any other activity in culture, society, and emotional values. Therefore, sexual desire affects all aspects of the individual's lives. ${ }^{13}$ Numerous fac- tors can influence the sexual function of postmenopausal women, which has been addressed in numerous studies. In general, factors affecting sexual function have been studied in three domains including physical, emotional, and social factors. Considering the fact that socio-economic and cultural factors can influence on a person's and society's perception of sex and sexual behaviors, this study was conducted to evaluate the sexual function of postmenopausal women, along with its effective factors.

\section{Methods}

\section{Study design}

The review was conducted according to the Preferred Reporting Items for Systematic Reviews and Meta-Analyses (PRISMA) guidelines. ${ }^{14,15}$ The review included English language papers published from January 1, 2000 to July 21, 2017 through searching in Google Scholar (Google Inc., Mountain View, CA, USA), PubMed (National Library of Medicine, Bethesda, MD, USA), Ovid (New York, NY, USA), ISI Web of Science (Thomson Reuters, New York, NY, USA), Science Direct (Elsevier, Amsterdam, Netherlands), Scopus (Elsevier), ProQuest (Ann Arbor, MI, USA), CINHAL (EBSCO, Ipswich, MA, USA), IranDoc (Iranian Research Institute for Information Science and Technology, Tehran, Iran) and SID (Scientific Information Database, Tehran, Iran).

\section{Search strategy}

The search key words included "menopause," "menopausal," "post-menopause," "post-menopausal," "pre-menapause," "pre-menopausal period," "sexual function," "sexual health," "sexuality," "sexual and gender disorders," "sexual development," "sexual dysfunction," "sexual disorders," "sexual behavior" and "sexual activity/ies." Using OR and AND, the key words were combined and entered in the search box of the databases as follows: (menopause OR menopausal OR post-menopause OR postmenopausal OR post-menopausal OR pre-menapause OR pre-menopausal period) AND (sexual function OR sexual health OR sexuality OR sexual and gender disorders OR sexual development OR sexual dysfunction OR sexual disorders OR sexual behavior OR sexual activity/ ies). 


\section{Evaluation of selected publications}

\section{(1) Inclusion/exclusion criteria}

Articles that investigated the sexual function and its effective factors in menopause and review of the original and review studies were considered as inclusion criteria. Also, studies that were not full paper available and before 2000 were excluded.

\section{Findings}

Figure 1 illustrates the process of study selection. After reviewing 1,312 articles, 27 articles including: 12 cross sectional, 7 descriptive, 3 prospective, 2 futuristic studies, 2 systematic reviews, and 1 qualitative study were selected.

\section{(1) Prevalence of sexual dysfunction}

In a study conducted by Dombek et al. ${ }^{16}$ the rate of sexual dysfunction was reported 70.3\% among postmenopausal women. In addition, Jonusiene et al. ${ }^{17}$ reported this rate as 67.9\% among postmenopausal women in Lithuania. In a sectional study done by Masliza et al., ${ }^{18}$ confirmed that the prevalence of sexual dysfunction in women was $85.2 \%$ in
Malaysia. Blümel et al. ${ }^{19}$ reported the prevalence of sexual dysfunction between $21 \%$ and $98.5 \%$ in their study, and this difference was related to the studied country and populations. In Iran, Kabudi, ${ }^{20}$ in his research on the knowledge about menopause and sexual function of women over 35 years old in Kermanshah, reported that sexual dysfunction responses in two phases of sexuality and erection in 141 menopausal women is $70 \%$. However, Yazdanpanahi et al. ${ }^{21}$ reported the prevalence of sexual dysfunction during menopause as much as 88.7\%. In another report in 2002 from the USA, this rate was $30 \%{ }^{22}$ Nazarpour et al. ${ }^{23}$ in their study aimed at examining the relationship between sexual function and the life quality of 405 Iranian postmenopausal women, determined that the average total score of sexual function was $24.11 \pm 1.10$. Further, the lowest and highest scores were related to erection and satisfaction, respectively. According to other studies, the prevalence of sexual dysfunction during menopause was achieved $51.3 \%$ in Chile, ${ }^{24}$ $40.4 \%$ in Nigeria, ${ }^{25}$ and $35.9 \%$ in Brazil. ${ }^{26}$ Furthermore, in a population-based study, which was conducted in 28 cities of Iran, it was estimated that 39\% of women over 50 years old are indulged with sexual dysfunction. ${ }^{27}$ In this regard,

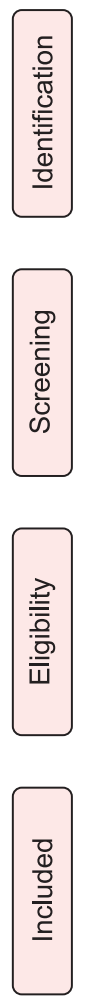

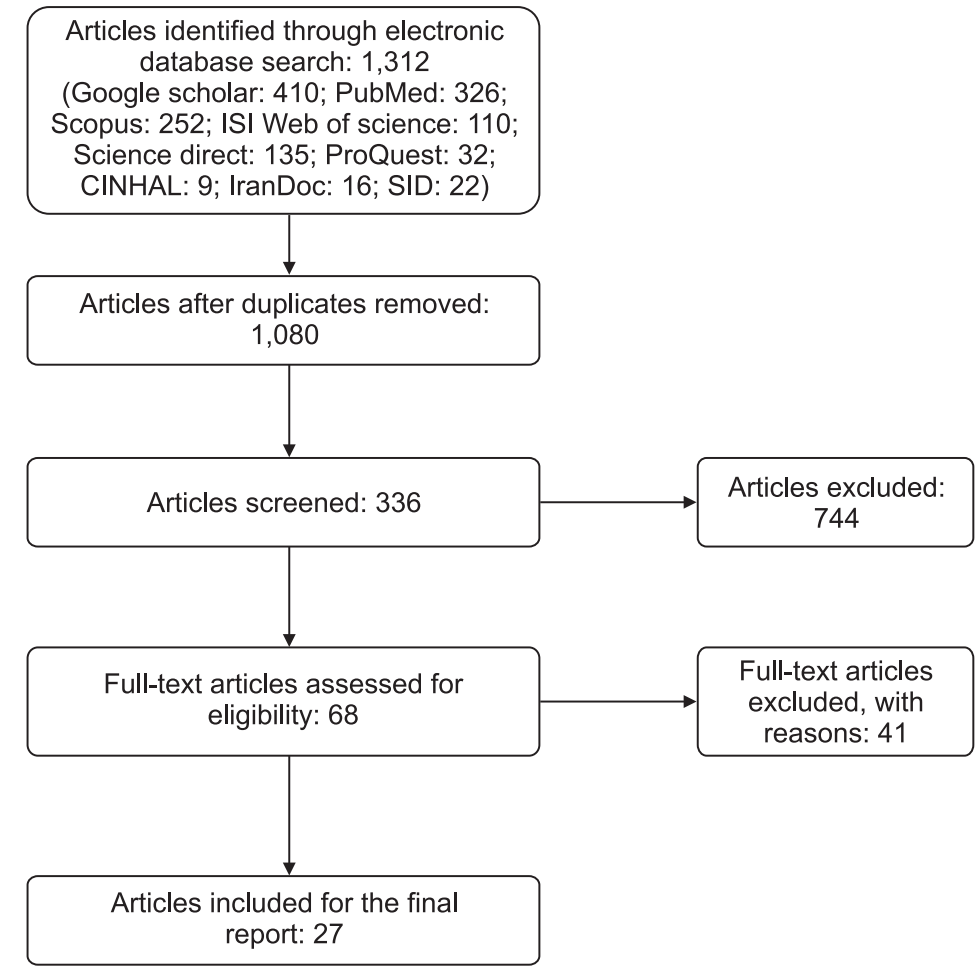

Fig. 1. Review selection process and results, in accordance with the the Preferred Reporting Items for Systematic Reviews and MetaAnalyses (PRISMA) guidelines. 
in a review study, da Silva Lara et al. ${ }^{28}$ found that $22 \%$ of postmenopausal women were involved in sexual activity only for the satisfaction of their husbands, and they were not willing to participate in these activities. Hashemi et al. ${ }^{29}$ in another cross-sectional study on 225 postmenopausal women between 45 and 65 years old in Iran, revealed that $70 \%$ of studied women at least are suffering from one sexual problem. Furthermore, in the study conducted by Topatan and Ylld $z^{30}$ on 450 postmenopausal women in Turkey, a positive relationship was observed between multiple sexual dysfunctions and the comprehended level of menopause symptoms' intensity. In this regard, Simon et al. ${ }^{31}$ in their study on 2000 postmenopausal women and men in North America, showed that vaginal discomfort leads to loss of sexual desire (64\%), and sexual pain (64\%), and almost 30\% of women and men introduced vaginal discomfort as a reason for stopping intercourse. In some studies, sexual complaints such as reduced sexual activity, decreased sexual response, difficulty in reaching to orgasm, and loss of genital sensation are associated with decreased estradiol levels. ${ }^{32}$ As mentioned, the factors affecting sexual function can be categorized in three domains: physical, emotional, and social. Sexual function of women after menopause is often influenced by vasomotor, neurogenic, endocrine, muscular, psychotic, drowsiness and symptoms of metabolic and psychological disorders. ${ }^{11}$

\section{(2) Demographic factors and sexual dysfunction}

By examining some studies, it was found that there is a negative correlation between sexual function and individual' s age. ${ }^{24,33}$ Other studies have reported positive association between poor sexual function and age. ${ }^{34,35}$ In addition, in some studies, a negative relationship was found between the age of the spouse and female sexual function. ${ }^{36}$ In the field of education, a number of studies have revealed a positive connection between the level of female education or sexual partner with sexual function. ${ }^{8,37,38}$ Beygi et al. ${ }^{39}$ considered spouse's education as an effective element in the sexual function of postmenopausal women. The study done by Dennerstein et al $^{40}$ confirmed the relationship between the education of couples and sexual dysfunction of women.

\section{(3) Androgens and sexual dysfunction}

About estrogen deprivation, there has been an agree- ment on the reduction of sexual function. ${ }^{41}$ Other studies reported that lower levels of testosterone can predict sexual dysfunction in postmenopausal women. ${ }^{42}$ In his review, Schwenkhagen $^{43}$ emphasized that reducing sexual desire, sexual stimulation, vaginal lubrication, etc., are associated with a decrease in the level of androgen. In another study, Gerber et al. ${ }^{33}$ conducted an interventional study on the effects of testosterone and psychological variables on the sexual function of postmenopausal women and indicated that there is a relationship between testosterone levels and sexual satisfaction between men 1 and 5 years after menopause. Further, Nappi et al. ${ }^{44}$ conducted a test on hormonal and psychological aspects and their association with sexual function during menopause and early stages of menopause. The results confirmed that sexual function was generally various at different stages of menopause. The application of hormone replacement therapy in a number of studies revealed positive impacts on the sexual function of postmenopausal women. ${ }^{45,46}$ However, in another study, hormone therapy was not considered to be effective in stimulation and desire. $^{45}$

\section{(4) Disorders and sexual dysfunction}

According to some studies, ovarian disorders and bladder surgeries were related to sexual dysfunction. ${ }^{16}$ Based on the results of the study by Nazarpour et al. ${ }^{23}$ the incidence of medical problems in a person or a spouse was suggested as a remarkable factor in the sexual function of menopausal women. Among the medical problems, cardiovascular diseases, diabetes, and musculoskeletal problems in postmenopausal women were accounted as predictive factors in the scores of some domains and the overall score of sexual function. ${ }^{23,47,48}$ Other studies have reported the connection between medical problems such as overactive bladder syndrome with sexual function in postmenopausal women. ${ }^{38,49}$ Obesity issue is regarded as one of the other medical problems. In another study, increased sexual activity was observed among women with lower body mass index. ${ }^{50}$ Other factors are related to physical activity. In this regard, the results of a study indicated that exercise is a variable which is significantly related to sexual satisfaction. ${ }^{33}$ 


\section{(5) Menopause and sexual dysfunction}

In a survey conducted by Guthrie et al., ${ }^{51}$ which lasted 9 years, it became clear that as the menopause's start delays, sexual abnormalities may increase from $42 \%$ to $88 \%$ due to a severe decrease of sexual hormones. The results of a review study revealed that overall sexual function decreased from $88 \%$ in the first year after menopause to $34 \%$ after 8 years. ${ }^{28}$ However, Blumel et al. ${ }^{52}$ reported that sexual function increases after five years of menopause which is related to a reduction in the severity of symptoms and a shift away from acute menopausal conditions. The reason for this contradiction may be due to the positive attitude that women in some societies gain toward menopause after menstruation. Another review indicated that low sexual function was positively associated to the number of pregnancies, deliveries, and abortions. ${ }^{35}$

\section{(6) Psycho-emotional and sexual dysfunction}

Among the factors studied in different studies, the psychoemotional field, depression, anxiety are highlighted. The findings of the studies reported that there is a significant relationship between sexual dysfunction, depression and anxiety. $^{17,39,53}$ Furthermore, they suggested that depression is the most important element in describing sexual dysfunction. ${ }^{21}$ Researchers believe that reducing sexual desire causes mental, emotional disorders and interpersonal distress, leading to a decline in general health. ${ }^{54}$

\section{(7) Culture, religion and attitude and sexual dysfunction}

Graziottin and Basson, ${ }^{55}$ in their study, reported that sexual behaviors are complex and diverse and are influenced by interpersonal relationships, lifestyle and cultural conditions. This behavior is closely related to the biological structure and the general feeling of the individual towards himself or herself as a man or a woman, and the experiences and developments of a person with respect to sexual matters. The underlying and interpersonal issues are responsible for most of the changes, which are experienced in sexual life during menopausal years, but they are not responsible for all of them. ${ }^{56}$ Further, social expectations have a negative impact on sexual desire. Some cultures believe that older women are diverted from sexuality. ${ }^{57}$ Nisar and Ahmed Sohoo ${ }^{58}$ demonstrated that postmenopausal women from traditional societies often tend to take care of children, grandchildren and religious practices during this time, and that participation in sexual activities is observed as later priority for them. Evidences from other investigations have suggested that the prevalence of sexual dysfunction is affected by racial, religious, cultural and attitudinal factors because social attitudes, cultural roles and religious believes can influence the sexual orientation of older women. ${ }^{8,34}$ García Padilla et al. ${ }^{59}$ proved that increased sexual dysfunction and other menopausal symptoms among these women are related to their low sexual awareness. Nappi and Nijland ${ }^{60}$ conducted a $^{2}$ study in Europe on women's understanding about gender in during menopause. They concluded that $34 \%$ of women were at risk of low libido.

\section{(8) Quality of marital relationships and sexual dysfunction}

In addition, a large number of studies emphasized a significant statistical relationship between the quality of the relationship with the spouse and sexual dysfunction. The results of another study by Beygi et al $^{39}$ revealed that the least amount of sexual dysfunctions was found in a group that reported very good relationship with their spouses. Accordingly, the percentage of sexual dysfunctions increased as the quality of couples' relationships in these groups reduced since $100 \%$ of the cases had sexual dysfunction in a group with a very bad relationship with their spouses.

\section{(9) Employment status and sexual dysfunction}

Furthermore, some studies proposed that employed women have a greater sexual desire than housewives, which can affect sexual satisfaction and is considered important in other studies. ${ }^{39,61}$ However, Blumel et al. ${ }^{52}$ found that housewives had a better quality of life in terms of sex than employed women, while employed women were more likely to be self-confident due to financial independence. ${ }^{62,63}$ The women with low income can have fewer sexual attraction, leading to a decrease in their sexual activities. ${ }^{35}$ Jokinen et al. ${ }^{64}$ focused on the occupation of women and sexual disorders. Another study suggests that women's age and marriage can reduce their sexual function. ${ }^{54}$ The duration of marriage and marriage are considered as other social factors. In another 


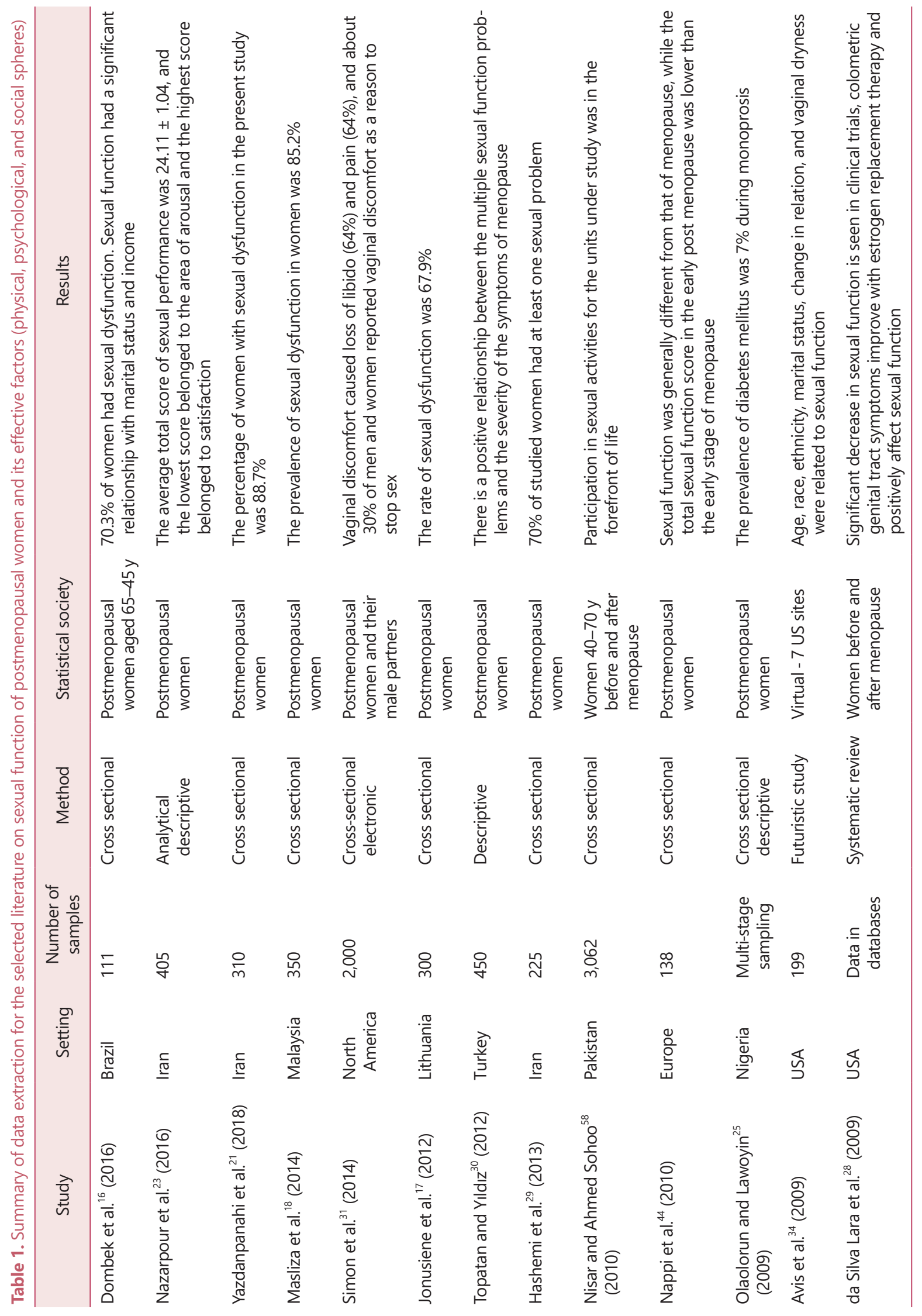




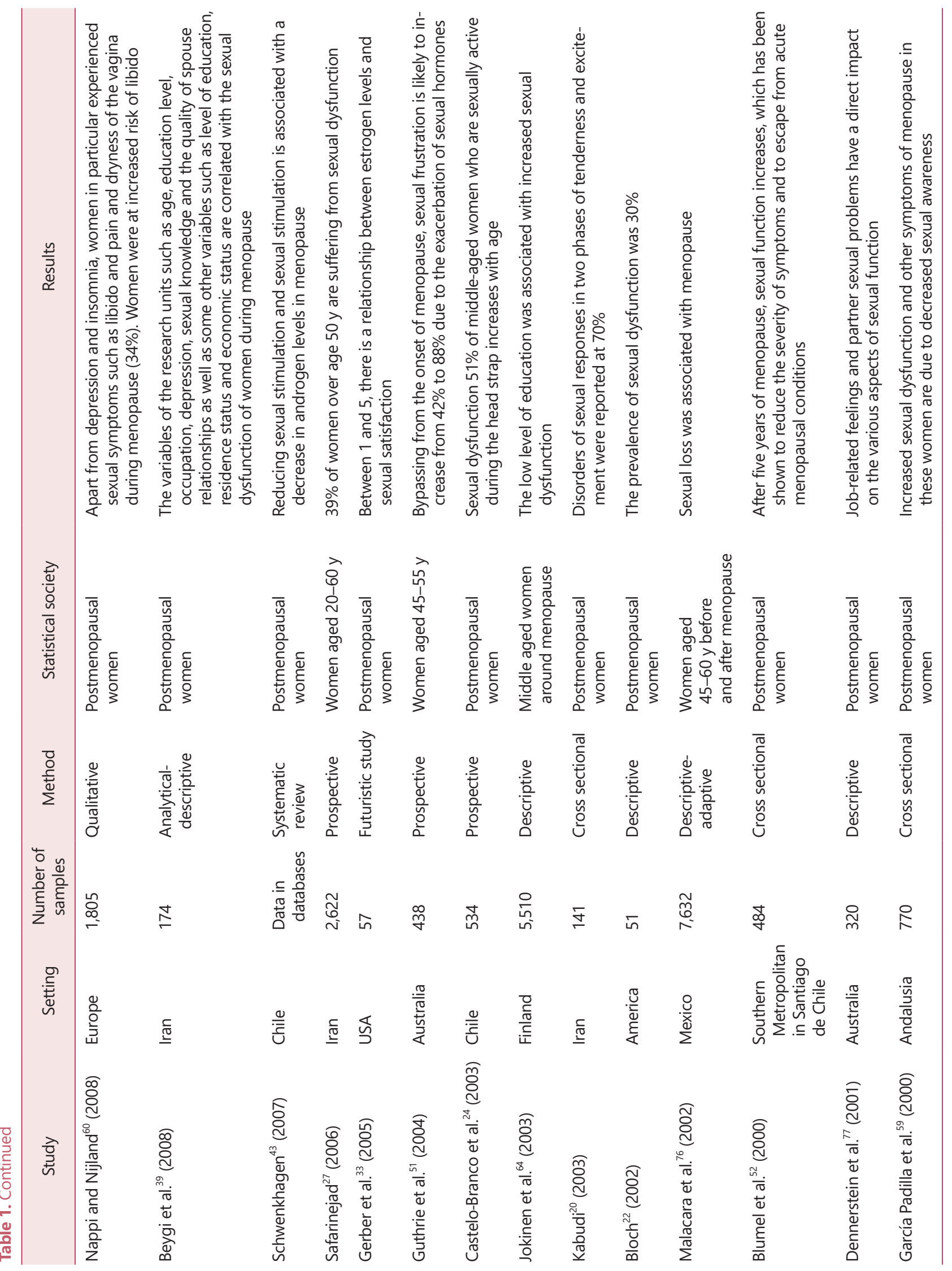


study, less problems were related to sexual intercourse by increase in life expectancy. ${ }^{65}$ In another study, the length of women's marriage could reduce their sexual function ${ }^{17}$ and the high risk of sexual dysfunction was significantly related to the duration of marriage. ${ }^{66}$ The findings of the studies conducted on sexual function and factors affecting the sexual functions of postmenopausal women (physical, mental and social domains) are presented in Table 1.

\section{Discussion}

In many countries around the world, the average life expectancy of women is about 80 years. Although the ovaries have lost their activity 25 years before reaching that age, menstrual bleeding does not continue until the age of 51 years on average. Thus, every woman is associated the last third part of her life with lack of production and deficiency of sex hormones. Symptoms of menopause are often due to the reduction of estrogen hormone, which can lead to complications such as hot flashes, sweating, headaches, muscle and joint aches, sleep disorders, etc., which may have an adverse effect on the quality of life of a person and the starting point for a series of side effects. For example, urogenital epithelia atrophy can lead to frequent urination, pain during urination, and discomfort in the vaginal area. ${ }^{67}$ Other symptoms of estrogen deficiency include depression, fatigue, redness and inflammation of the skin, memory disorders, lack of concentration and loss sexual desire. ${ }^{68}$ Therefore, the proposed studies have considered the effect of estrogen deprivation in reducing sexual function. ${ }^{33,42,43}$ Regarding physical activities such as exercise, Lange-Collett ${ }^{69}$ argued that exercise and nutrition are two important factors in the life of postmenopausal women and determined their health status. In another study, Verit et al. ${ }^{35}$ reported exercise as an alternative treatment option for reducing menopausal symptoms such as psychological, mediational, physical, and sexual symptoms. In the same study, the effect of regular exercise on the improvement of sexual function was highlighted. ${ }^{33}$ Based on the results of some studies, it seems that the presence of medical condition is regarded as one of the outstanding issues which can influence sexual function. ${ }^{23,38,49,50}$ Chronic medical conditions tend to decrease simulation and desire. In this regard, Nascimento et al. ${ }^{70}$ in their systematic review about sexual dysfunction and cardiovascular diseases, reported that in all women, all areas of sexual functions such as tenderness, simulation, lubrication, orgasm, sexual satisfaction and pain are affected by cardiovascular diseases and cardiovascular disease are risk factors for developing sexual dysfunction in women and men. The severity of heart diseases increases the incidence of sexual dysfunction. In addition, the psychosocial factors originated from cardiovascular events gradually affected the sexual life of patients which contribute to sexual dysfunctions. Further, symptoms of depression associated with cardiovascular diseases lead to impaired sexual response. In an experiment by Doruk et al. ${ }^{71}$ the scores of sexual desire, stimulation and lubrication in the type I diabetic group were significantly lower than in the control group. However, no risk factor was discovered which could predict sexual dysfunction in diabetic women. Postmenopausal women experience numerous psychological changes and have feelings about their negative reactions to menopausal transformations. These changes can affect interpersonal, social, family, and the total quality of their life. ${ }^{72}$ The reduction of sexual function is considered as one of the important complications of this period which play a negative effect on their sexual function according to the reviewed articles. ${ }^{17-19}$ Additionally, some studies indicated that postmenopausal women experience aging, altered mental image of their appearance, and feelings of femininity end, disability, disappointment, depression, and anxiety which can influence their sexual function. ${ }^{72}$ In line with the results of some control studies, ${ }^{17,21,39,53}$ Borissova et al. ${ }^{73}$ reported that decreased libido in postmenopausal women may be related to some psychological factors like depression. Danaci et al. ${ }^{74}$ indicated that anxiety and depression have a profound effect on sexual relationships. Some experiments emphasized the relationship between age and sexual function. ${ }^{24,33-36}$ An increase in age leads to a decrease in sexual responses and sexual desire, as well as the frequency of sexual intercourse during menopause. Consequently, sexual function faces with disorder which can be an important factor in reducing sexual function in postmenopausal women. ${ }^{75}$ In addition, Malacara et al., ${ }^{76}$ in another study on Mexican women with urban and rural settlements, reported that libido was affected by aging so that postmenopausal women 
tended to have reduced sexual desire more than women before menopause. Dennerstein et al. ${ }^{77}$ demonstrated that women's sexual responses during middle age are influenced by two factors of age, while Gott and Hinchliff ${ }^{78}$ reported that aging and long-term relationships with the spouse are needed to counteract the decline in couples' sexual desires. Ponholzer et al. ${ }^{79}$ in their study on the risk factors and the prevalence of sexual dysfunction among 703 Australian women, found that $22 \%, 35 \%$, and $39 \%$ had sexual desire dysfunctions, sexual arousal, and orgasmic problems, respectively, and all of these factors increased significantly by aging. Similarly, in another study, on low sexual desire in middle-aged and old women, Hartmann et al. ${ }^{80}$ found similar results by indicating the prevalence of sexual dysfunction, especially low sexual desire and sexual arousal disorders with aging. The stressors in life, the underlying factors such as the quality of communication and personality factors, past experiences and sexual desires, and physical and mental health are other important predictors of female sexual dysfunction in this study. The level of education is a predictive variable for psychological complications and negative emotions of menopause which can affect women's sexual function during this period, $8,37,38,40$ and it is regarded as one of the reasons that education increases self-confidence and helps to have a good impression of health. ${ }^{81}$ The results of some studies revealed that female sexual function is reduced by increasing the level of education. ${ }^{82,83}$ On the other hand, the study on the reviewed articles confirmed the relationship between sexual performance and the quality of the relationship with the spouse. ${ }^{39}$ In another study conducted by Liu and Eden, ${ }^{84}$ many Chinese postmenopausal women believed that a decline in sexual activity begins with menopause and if their husbands were willing to remarry due to disrupted sexual relationship, they would agree on this because they believed that during this period, lack of libido may lead to separation and more emotional distance with their husbands. In addition, Beutel et al. ${ }^{85}$ reported old German men consider proper relationships with their wives as a factor in increasing sexual desire. In this regard, Kingsberg ${ }^{86}$ proved that the mental or physical problems of the sexual partner and, consequently, the reduction of the desired relationship with the partner are key factors for developing sexual dysfunctions. Further, having a good relationship between the couples during middle and old age decreases the dramatic effect of their physical and psychological statues of these individuals on their sexual relationship and these individuals can follow normal sexual activities. The prevalence of sexual dysfunctions varies from country to country. Considerable differences among the statistics can be attributed to racial, religious, cultural and attitudinal factors, because social attitude, cultural roles and religious believes can affect the experience of sexual desires of elderly women, ${ }^{87}$ as it was highlighted by a large number of studies. ${ }^{8,34,58}$ Wright $^{88}$ believes that female sexual value and attraction are only for reproduction in some cultures, while Deacon et al. ${ }^{89}$ emphasize that women are less important than the women at the age of fertility in terms of sexual value due to lack of reproduction and it seems to be more likely as the result of community culture. This is justified by the fact that having a high income is likely to lead to more mental relief, which leads to sexual satisfaction. The middle age is a period of attention to spirituality, and people often evaluate themselves and have a special look at the end of their lives. The type of religious attitude is important to sexual relationships and can affect sexual satisfaction. ${ }^{87}$ Findings confirm the relationship between sexual function and awareness of postmenopausal women. ${ }^{59,60}$ Knowledge of menopause trend can increase orgasmicity. High awareness which can be achieved through higher education can change attitudes and thoughts toward gender, reduce anxiety, and helps the individual adapt to menopause. ${ }^{90}$ In another review conducted on Chinese women, Beutel et al. ${ }^{85}$ concluded that they had no information about treatments which can eliminate sexual dysfunction and their awareness was very low. Increasing sexual awareness during this period helps the individuals not to consider the sexual dysfunctions abnormal which are resulted from menopausal symptoms and changes in sexual behavior by aging, but attempted to adapt, resolve or mitigate these problems. Therefore, the role of sexual awareness in preventing and treating sexual disorders in women is quite obvious. It seems embarrassment to talk about sexual issues, the lack of proper understanding of sexual issues, and lack of sufficient information about these issues are regarded as some significant causes of sexual problems in the early experiences of married life. ${ }^{66}$ Palacios et al. ${ }^{91}$ found that examining the sexual history of postmenopausal wom- 
en since fertility age is the first step in diagnosing and treating postmenopausal women with sexual dysfunction, which is consistent with the results of the studies which correlated sexual function with the number of pregnancies and childbirth. ${ }^{35}$ Regarding hormonal treatment, it seems that the hormone administration and its onset are effective in the level of sexual function. ${ }^{92}$ As it was already mentioned, some studies have shown the protective effect of hormone therapy on sexual function. ${ }^{45,46}$

\section{Conclusion}

Regarding all of the results obtained from this study, it is recommended that the staff of health centers should remind menopausal women about their evolution by aging and reaching menopause. In addition, they should emphasize sexual desire in every person's life by contemplated programming for the continuous and sequential training courses for menopausal women about sexual activity, the physiological changes during menopause, and their way of adapting themselves in order to prevent them from psychological and social consequences of sexual dysfunctions.

\section{Conflict of Interest}

No potential conflict of interest relevant to this article was reported.

\section{References}

1. Heidari M, Ghodusi M, Rafiei H. Sexual self-concept and its relationship to depression, stress and anxiety in postmenopausal women. J Menopausal Med 2017; 23: 42-8.

2. Heidari M, Shahbazi S, Ghafourifard M, Ali Sheikhi R. Prediction of marital satisfaction based on emotional intelligence in postmenopausal women. J Menopausal Med 2017; 23: 196-201.

3. Shirvani M, Heidari M. Quality of life in postmenopausal female members and non-members of the elderly support association. J Menopausal Med 2016; 22: 154-60.

4. Kim TH, Lee HH. Alternative therapy trends among Korean postmenopausal women. J Menopausal Med 2016; 22: 45.

5. Jafari M, Seifi B, Heidari M. Risk assessment: factors contributing to discomfort for menopausal women in workplace. J Menopausal Med 2017; 23: 85-90.

6. Enkhbold T, Jadambaa Z, Kim TH. Management of menopausal symptoms in Mongolia. J Menopausal Med 2016; 22 : $55-8$.

7. Thompson WK, Charo L, Vahia IV, Depp C, Allison M, Jeste DV. Association between higher levels of sexual function, activity, and satisfaction and self-rated successful aging in older postmenopausal women. J Am Geriatr Soc 2011; 59: 1503-8.

8. Ornat L, Martínez-Dearth R, Muñoz A, Franco P, Alonso B, Tajada M, et al. Sexual function, satisfaction with life and menopausal symptoms in middle-aged women. Maturitas 2013; 75: 261-9.

9. Chedraui P, Pérez-López FR, Sánchez H, Aguirre W, Martínez N, Miranda O, et al. Assessment of sexual function of mid-aged Ecuadorian women with the 6-item Female Sexual Function Index. Maturitas 2012; 71: 407-12.

10. Cabral PU, Canário AC, Spyrides MH, Uchôa SA, Eleutério $\mathrm{J} \mathrm{Jr}$, Amaral RL, et al. Influence of menopausal symptoms on sexual function in middle-aged women. Rev Bras Ginecol Obstet 2012; 34: 329-34.

11. Berman JR. Physiology of female sexual function and dysfunction. Int J Impot Res 2005; 17 Suppl 1: S44-51.

12. Rosen R, Brown C, Heiman J, Leiblum S, Meston C, Shabsigh $\mathrm{R}$, et al. The Female Sexual Function Index (FSFI): a multidimensional self-report instrument for the assessment of female sexual function. J Sex Marital Ther 2000; 26: 191208.

13. Cheng MH, Wang SJ, Wang PH, Fuh JL. Attitudes toward menopause among middle-aged women: a community survey in an island of Taiwan. Maturitas 2005; 52: 348-55.

14. Moher D, Shamseer L, Clarke M, Ghersi D, Liberati A, Petticrew M, et al. Preferred reporting items for systematic review and meta-analysis protocols (PRISMA-P) 2015 statement. Syst Rev 2015; 4: 1.

15. Liberati A, Altman DG, Tetzlaff J, Mulrow C, Gøtzsche PC, Ioannidis JP, et al. The PRISMA statement for reporting systematic reviews and meta-analyses of studies that evaluate health care interventions: explanation and elaboration. PLoS Med 2009; 6: e1000100.

16. Dombek K, Capistrano EJ, Costa AC, Marinheiro LP. Risk factors associated with sexual dysfunction in Brazilian postmenopausal women. Int J Impot Res 2016; 28: 62-7.

17. Jonusiene G, Zilaitiene B, Adomaitiene V, Aniuliene R, Bancroft J. Sexual function, mood and menopause symptoms in Lithuanian postmenopausal women. Climacteric 
2012; 16: 185-93.

18. Masliza W, Daud W, Yazid Bajuri M, Shuhaila A, Hatta S, Rohaizat Hassan M, et al. Sexual dysfunction among postmenopausal women. Clin Ter 2014; 165: 83-9.

19. Blümel JE, Chedraui P, Baron G, Belzares E, Bencosme A, Calle A, et al. Sexual dysfunction in middle-aged women: a multicenter Latin American study using the Female Sexual Function Index. Menopause 2009; 16: 1139-48.

20. Kabudi M. The study of over 35 women's knowledge and interaction against menopause. Paper presented at: 1st Congress of Family and Sexual Disorders, Shahed University of Medical Sciences; 2003; Tehran, Iran.

21. Yazdanpanahi Z, Nikkholgh M, Akbarzadeh M, Pourahmad S. Stress, anxiety, depression, and sexual dysfunction among postmenopausal women in Shiraz, Iran, 2015. J Family Community Med 2018; 25: 82-7.

22. Bloch A. Self-awareness during the menopause. Maturitas 2002; 41: 61-8.

23. Nazarpour S, Simbar M, Ramezani Tehrani F, Alavi Majd H. Relationship between sexual function and quality of life in post-menopausal women. J Mazandaran Univ Med Sci 2016; 26: $90-8$.

24. Castelo-Branco C, Blumel JE, Araya H, Riquelme R, Castro G, Haya J, et al. Prevalence of sexual dysfunction in a cohort of middle-aged women: influences of menopause and hormone replacement therapy. J Obstet Gynaecol 2003; 23: 426-30.

25. Olaolorun FM, Lawoyin TO. Experience of menopausal symptoms by women in an urban community in Ibadan, Nigeria. Menopause 2009; 16: 822-30.

26. Valadares AL, Pinto-Neto AM, Osis MJ, Sousa MH, CostaPaiva L, Conde DM. Prevalence of sexual dysfunction and its associated factors in women aged 40-65 years with 11 years or more of formal education: a population-based household survey. Clinics (Sao Paulo) 2008; 63: 775-82.

27. Safarinejad MR. Female sexual dysfunction in a populationbased study in Iran: prevalence and associated risk factors. Int J Impot Res 2006; 18: 382-95.

28. da Silva Lara LA, Useche B, Rosa E Silva JC, Ferriani RA, Reis RM, de Sá MF, et al. Sexuality during the climacteric period. Maturitas 2009; 62: 127-33.

29. Hashemi S, Ramezani Tehrani F, Simbar M, Abedini M, Bahreinian H, Gholami R. Evaluation of sexual attitude and sexual function in menopausal age; a population based cross-sectional study. Iran J Reprod Med 2013; 11: 631-6.

30. Topatan S, Ylldz H. Symptoms experienced by women who enter into natural and surgical menopause and their relation to sexual functions. Health Care Women Int 2012; 33: 525-39.
31. Simon JA, Nappi RE, Kingsberg SA, Maamari R, Brown V. Clarifying Vaginal Atrophy's Impact on Sex and Relationships (CLOSER) survey: emotional and physical impact of vaginal discomfort on North American postmenopausal women and their partners. Menopause 2014; 21: 137-42.

32. Gallicchio L, Schilling C, Tomic D, Miller SR, Zacur H, Flaws JA. Correlates of sexual functioning among mid-life women. Climacteric 2007; 10: 13242.

33. Gerber JR, Johnson JV, Bunn JY, O’Brien SL. A longitudinal study of the effects of free testosterone and other psychosocial variables on sexual function during the natural traverse of menopause. Fertil Steril 2005; 83: 643-8.

34. Avis NE, Brockwell S, Randolph JF Jr, Shen S, Cain VS, Ory $\mathrm{M}$, et al. Longitudinal changes in sexual functioning as women transition through menopause: results from the Study of Women's Health Across the Nation. Menopause 2009; 16: 442-52.

35. Verit FF, Verit A, Billurcu N. Low sexual function and its associated risk factors in pre- and postmenopausal women without clinically significant depression. Maturitas 2009; 64: $38-42$.

36. Chedraui P, Pérez-López FR, Mezones-Holguin E, San Miguel G, Avila C; Collaborative Group for Research of the Climacteric in Latin America (REDLINC). Assessing predictors of sexual function in mid-aged sexually active women. Maturitas 2011; 68: 387-90.

37. Llaneza P, Fernández-Iñarrea JM, Arnott B, García-Portilla MP, Chedraui P, Pérez-López FR. Sexual function assessment in postmenopausal women with the 14-item changes in sexual functioning questionnaire. J Sex Med 2011; 8: $2144-51$.

38. Merghati-Khoei E, Sheikhan F, Shamsalizadeh N, Haghani H, Yousofnia Pasha YR, Killeen T. Menopause negatively impacts sexual lives of middle-aged Iranian women: a crosssectional study. J Sex Marital Ther 2014; 40: 552-60.

39. Beygi M, Fahami F, Hasan-Zahraei R, Arman S. Sexual dysfunction in menopause. J Isfahan Med Sch 2008; 26: 294-300.

40. Dennerstein L, Lehert P, Burger H, Dudley E. Factors affecting sexual functioning of women in the mid-life years. Climacteric 1999; 2: 254-62

41. Tansupswatdikul P, Chaikittisilpa S, Jaimchariyatam N, Panyakhamlerd K, Jaisamrarn U, Taechakraichana N. Effects of estrogen therapy on postmenopausal sleep quality regardless of vasomotor symptoms: a randomized trial. Climacteric 2015; 18: 198-204.

42. Mostafa T, Mostafa RM, Hassan MM, Nasrallah YS, Salman WS, Taymour M. Female sexual dysfunction among menopausal women. Hum Androl 2015; 5: 23-7. 
43. Schwenkhagen A. Hormonal changes in menopause and implications on sexual health. J Sex Med 2007; 4 Suppl 3: $220-6$.

44. Nappi RE, Albani F, Santamaria V, Tonani S, Magri F, Martini E, et al. Hormonal and psycho-relational aspects of sexual function during menopausal transition and at early menopause. Maturitas 2010; 67: 78-83.

45. González M, Viáfara G, Caba F, Molina E. Sexual function, menopause and hormone replacement therapy (HRT). Maturitas 2004; 48: 411-20.

46. Nastri CO, Lara LA, Ferriani RA, Rosa-E-Silva AC, Figueiredo JB, Martins WP. Hormone therapy for sexual function in perimenopausal and postmenopausal women. Cochrane Database Syst Rev 2013; (6): CD009672.

47. Heidari M, Shahbazi S, Ghodusi M. Evaluation of body esteem and mental health in patients with breast cancer after mastectomy. J Midlife Health 2015; 6: 173-7.

48. Heidari M, Ghodusi M. The relationship between body esteem and hope and mental health in breast cancer patients after mastectomy. Indian J Palliat Care 2015; 21: 198-202.

49. Hakimi S, Aminian E, Alizadeh Charandabi SM, Bastani P, Mohammadi M. Risk factors of overactive bladder syndrome and its relation to sexual function in menopausal women. Urologia 2018; 85: 104.

50. Park YJ, Kim HS, Chang SO, Kang HC, Chun SH. Sexuality and related factors of postmenopausal Korean women. Taehan Kanho Hakhoe Chi 2003; 33: 457-63.

51. Guthrie JR, Dennerstein L, Taffe JR, Lehert P, Burger HG. The menopausal transition: a 9-year prospective populationbased study. The Melbourne Women's Midlife Health Project. Climacteric 2004; 7: 375-89.

52. Blumel JE, Castelo-Branco C, Binfa L, Gramegna G, Tacla X, Aracena B, et al. Quality of life after the menopause: a population study. Maturitas 2000; 34: 17-23.

53. Mezones-Holguin E, Córdova-Marcelo W, Lau-Chu-Fon F, Aguilar-Silva C, Morales-Cabrera J, Bolaños-Díaz R, et al. Association between sexual function and depression in sexually active, mid-aged, Peruvian women. Climacteric 2011; 14: 654-60.

54. Yangin HB, Sözer GA, Sengün N, Kukulu K. The relationship between depression and sexual function in menopause period. Maturitas 2008; 61: 233-7.

55. Graziottin A, Basson R. Sexual dysfunction in women with premature menopause. Menopause 2004; 11: 766-77.

56. Bachmann GA, Leiblum SR. The impact of hormones on menopausal sexuality: a literature review. Menopause 2004; 11: $120-30$.

57. Omu AE, al-Qattan N. Effects of hormone replacement therapy on sexuality in postmenopausal women in a mid- east country. J Obstet Gynaecol Res 1997; 23: 157-64.

58. Nisar N, Ahmed Sohoo N. Severity of Menopausal symptoms and the quality of life at different status of Menopause: a community based survey from rural Sindh, Pakistan. Int J Collab Res Intern Med Public Health 2010; 2: 118 30.

59. García Padilla FM, López Santos V, Toronjo Gómez AM, Toscano Márquez T, Contreras Martín A. Evaluation of knowledge about climacteric in Andalusian women. Aten Primaria 2000; 26: 476-81.

60. Nappi RE, Nijland EA. Women's perception of sexuality around the menopause: outcomes of a European telephone survey. Eur J Obstet Gynecol Reprod Biol 2008; 137: 10-6.

61. da Fonseca AM, Cavalcanti AL, Bagnoli VR, da Paixão JS, Penteado SRL, Cardoso EB, et al. Effects of estradiol valerate associated with cyproterone acetate on some clinical and sexuality-related aspects in postmenopausal women. Einstein 2008; 6: 170-4.

62. Osinowo HO. Psychosocial factors associated with perceived psychological health, perception of menopause and sexual satisfaction in menopausal women and controls. West Afr J Med 2003; 22: 225-31.

63. Elavsky S, McAuley E. Physical activity, symptoms, esteem, and life satisfaction during menopause. Maturitas 2005; 52: 374-85.

64. Jokinen K, Rautava P, Mäkinen J, Ojanlatva A, Sundell J, Helenius H. Experience of climacteric symptoms among 4246 and 52-56-year-old women. Maturitas 2003; 46: 199-205.

65. Ishak IH, Low WY, Othman S. Prevalence, risk factors, and predictors of female sexual dysfunction in a primary care setting: a survey finding. J Sex Med 2010; 7: 3080-7.

66. Bolourian Z, Ganjloo J. Evaluating sexual dysfunction and some related factors in women attending Sabzevar Health Care Centers. J Reprod Infertil 2007; 8: 163-70.

67. Brynhildsen J, Hammar M. Low dose transdermal estradiol/norethisterone acetate treatment over 2 years does not cause endometrial proliferation in postmenopausal women. Menopause 2002; 9: 137-44.

68. Shumaker SA, Legault C, Rapp SR, Thal L, Wallace RB, Ockene JK, et al. Estrogen plus progestin and the incidence of dementia and mild cognitive impairment in postmenopausal women: the Women's Health Initiative Memory Study: a randomized controlled trial. JAMA 2003; 289: 265162.

69. Lange-Collett J. Promoting health among perimenopausal women through diet and exercise. J Am Acad Nurse Pract 2002; 14: 172-9.

70. Nascimento ER, Maia AC, Pereira V, Soares-Filho G, Nardi AE, Silva AC. Sexual dysfunction and cardiovascular dis- 
eases: a systematic review of prevalence. Clinics (Sao Paulo) 2013; 68: 1462-8.

71. Doruk H, Akbay E, Cayan S, Akbay E, Bozlu M, Acar D. Effect of diabetes mellitus on female sexual function and risk factors. Arch Androl 2005; 51: $1-6$.

72. Afghari A, Ganji J, Ahmad Shirvani M. Psycho-emotional changes in menopause: a qualitative study. J Mazandaran Univ Med Sci 2012; 22: 27-38.

73. Borissova AM, Kovatcheva R, Shinkov A, Vukov M. A study of the psychological status and sexuality in middle-aged Bulgarian women: significance of the hormone replacement therapy (HRT). Maturitas 2001; 39: 177-83.

74. Danaci AE, Oruç S, Adigüzel H, Yildirim Y, Aydemir O. Relationship of sexuality with psychological and hormonal features in the menopausal period. West Indian Med J 2003; 52: 27-30.

75. Basson R. Women's sexual dysfunction: revised and expanded definitions. CMAJ 2005; 172: 1327-33.

76. Malacara JM, Canto de Cetina T, Bassol S, González N, Cacique L, Vera-Ramírez ML, et al. Symptoms at pre- and postmenopause in rural and urban women from three States of Mexico. Maturitas 2002; 43: 11-9.

77. Dennerstein L, Dudley E, Burger H. Are changes in sexual functioning during midlife due to aging or menopause? Fertil Steril 2001; 76: 456-60.

78. Gott M, Hinchliff S. How important is sex in later life? The views of older people. Soc Sci Med 2003; 56: 1617-28.

79. Ponholzer A, Roehlich M, Racz U, Temml C, Madersbacher S. Female sexual dysfunction in a healthy Austrian cohort: prevalence and risk factors. Eur Urol 2005; 47: 366-75.

80. Hartmann U, Philippsohn S, Heiser K, Rüffer-Hesse C. Low sexual desire in midlife and older women: personality factors, psychosocial development, present sexuality. Menopause 2004; 11: 726-40.

81. Pérez JA, Garcia FC, Palacios S, Pérez M. Epidemiology of risk factors and symptoms associated with menopause in Spanish women. Maturitas 2009; 62: 30-6.

82. Tomic D, Gallicchio L, Whiteman MK, Lewis LM, Langenberg P, Flaws JA. Factors associated with determinants of sexual functioning in midlife women. Maturitas 2006; 53 : 144-57.

83. Addis IB, Van Den Eeden SK, Wassel-Fyr CL, Vittinghoff E, Brown JS, Thom DH. Sexual activity and function in middleaged and older women. Obstet Gynecol 2006; 107: 755-64.

84. Liu J, Eden J. Experience and attitudes toward menopause in Chinese women living in Sydney-a cross sectional survey. Maturitas 2007; 58: 359-65.

85. Beutel ME, Schumacher J, Weidner W, Brähler E. Sexual activity, sexual and partnership satisfaction in ageing men-results from a German representative community study. Andrologia 2002; 34: 22-8.

86. Kingsberg SA. The impact of aging on sexual function in women and their partners. Arch Sex Behav 2002; 31: 431-7.

87. Lin TB. Sexuality and the menopause. Paper presented at: First Consensus Meeting on Menopause in the East Asian Region; 2013; Geneva, Swiss.

88. Wright LK. Affection and sexuality in the presence of Alzheimer's disease: a longitudinal study. Sex Disabil 1998; 16: 167-79.

89. Deacon S, Minichiello V, Plummer D. Sexuality and older people: revisiting the assumptions. Educ Gerontol 1995; 21: 497-513.

90. Meston CM, Levin RJ, Sipski ML, Hull EM, Heiman JR. Women's orgasm. Annu Rev Sex Res 2004; 15: 173-257.

91. Palacios S, Tobar AC, Menendez C. Sexuality in the climacteric years. Maturitas 2002; 43 Suppl 1: S69-77.

92. Alarslan D, Sarandol A, Cengiz C, Develioglu OH. Androgens and sexual dysfunction in naturally and surgically menopausal women. J Obstet Gynaecol Res 2011; 37: 102734. 